\title{
Dopant-stress synergy in Si solid-phase epitaxy
}

\author{
N. G. Rudawski, ${ }^{1, a)}$ K. S. Jones, ${ }^{1}$ and R. Gwilliam ${ }^{2}$ \\ ${ }^{1}$ Department of Materials Science and Engineering, University of Florida, \\ Gainesville, Florida 32611-6400, USA \\ ${ }^{2}$ Nodus Accelerator Laboratory, Advanced Technology Institute, Surrey Ion Beam Centre, Guildford, \\ Surrey GU2 7XH, United Kingdom
}

(Received 29 April 2008; accepted 26 May 2008; published online 12 June 2008)

The influence of dopants on stressed solid-phase epitaxy of Si was studied in B-doped material up to B concentration of $\sim 3.0 \times 10^{20} \mathrm{~cm}^{-3}$ and stress of $1.0 \pm 0.1 \mathrm{GPa}$. As per the generalized Fermi level shifting model of growth enhancement in the presence of electrically active impurities, it is advanced that application of compressive stress may increase the energy difference between intrinsic Fermi and acceptor levels thus making dopant and stress effects synergistic in growth kinetics. (C) 2008 American Institute of Physics. [DOI: 10.1063/1.2945291]

Solid-phase epitaxial growth (SPEG) of amorphized Si is a topic of great technological importance. ${ }^{1}$ In particular, there is interest in studying the roles of electrically active impurities and, more recently, stress states $\left(\sigma_{i j}\right)$ on growth kinetics due the prevalent nature of stresses in $\mathrm{Si}$ device processing. ${ }^{2}$ At light electrically active impurity levels, the velocity $\nu$ of an advancing growth front between amorphous $(\alpha)$ and crystalline phases can be modeled as linearly enhanced with dopant concentration stemming from a shift in the Fermi energy at the $\alpha /$ crystalline (growth) interface. ${ }^{3-6}$ This is known as the generalized Fermi level shifting (GFLS) model. In the case of B-doped $\mathrm{Si}, \nu$ takes the form

$$
\nu=\nu_{i}\left[1+\left(C_{\mathrm{B}} g / n_{i}\right) \exp \left(-\frac{E_{F}^{i}-E_{n}^{p}}{k T}\right)\right],
$$

where $k T$ has the usual meaning, $\nu_{i}$ is the intrinsic SPEG velocity at $T, C_{\mathrm{B}}$ is the electrically active $\mathrm{B}$ concentration at the growth interface, $g$ is the degeneracy factor $(1.5 \pm 0.2), n_{i}$ is the intrinsic carrier concentration ${ }^{7}$ at $T, E_{F}^{i}$ is the intrinsic Fermi level $(0.55 \mathrm{eV})$, and $E_{n}^{p}$ is the acceptor energy level for B $(0.20 \pm 0.02 \mathrm{eV})$. By convention, all energy levels are measured relative to the top of the valence band. ${ }^{6}$

From an atomistic standpoint, growth results from crystal island nucleation at the growth interface with subsequent in-plane migration of island ledges. ${ }^{8,9}$ The GFLS model proposes that individual nuclei may possess charge states such that the total nucleation rate (with $\sigma_{i j}=0$ ) is given by $\tau_{n}(0)^{-1}=\tau_{n}^{j}(0)^{-1}+\tau_{n}^{p}(0)^{-1}$, where $\tau_{n}^{j}(0)^{-1}$ and $\tau_{n}^{p}(0)^{-1}$ are the stress-free nucleation rates of uncharged and positively charged nuclei in $p$-type material. Thus, in the case of charged nuclei, nucleation kinetics will be Fermi level dependent. Assuming a Maxwell-Boltzmann approximation (reasonable for dilute dopant levels) and using the usual expression for Fermi level as a function of doping, it can be shown that

$$
\tau_{n}(0)^{-1}=\tau_{n}^{i}(0)^{-1}\left[1+\left(C_{\mathrm{B}} g / n_{i}\right) \exp \left(-\frac{E_{F}^{i}-E_{n}^{p}}{k T}\right)\right]
$$

in B-doped $\mathrm{Si}^{10}{ }^{10}$ In conjunction with recent work ${ }^{11,12}$ where the stress-free growth velocity was shown to be given by

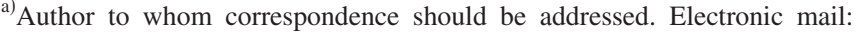
ngr@ufl.edu. $\nu=2 \Delta x \tau_{n}(0)^{-1}$, where $\Delta x$ is the monolayer spacing $(0.14 \mathrm{~nm})$, Eq. (1) may be rewritten as

$$
\nu=\frac{2 \Delta x}{\tau_{n}^{i}(0)}\left[1+\left(C_{\mathrm{B}} g / n_{i}\right) \exp \left(-\frac{E_{F}^{i}-E_{n}^{p}}{k T}\right)\right] .
$$

In terms of stressed-SPEG, recent work in intrinsic (001) $\mathrm{Si}$ advanced $\nu$ as a function of uniaxial stress in the plane of the growth front $\left(\sigma_{11}\right)$ of the form

$$
\begin{aligned}
\nu= & \frac{\Delta x}{\tau_{n}^{i}(0)+2^{-3 / 2} \tau_{m, 11}^{j}(0) \exp \left(\frac{-\Delta V_{11}^{m, 11} \sigma_{11}}{k T}\right)} \\
& +\frac{\Delta x}{\tau_{n}^{i}(0)+2^{-3 / 2} \tau_{m, 11}^{i}(0)},
\end{aligned}
$$

where $\tau_{m, 11}^{i}(0)$ is the intrinsic $\sigma_{i j}=0$ time for ledge migration along 1 and $\Delta V_{11}^{m, 11}$ is the activation volume for ledge migration along 1 in the 1 direction. By convention, 1 and 2 are the in-plane directions, 3 is the growth direction, and positive (negative) elements of $\sigma_{i j}$ are tensile (compressive). A consequence of Eq. (4) is that $\nu$ has finite limits. In the case of $0 \leqslant \sigma_{11}, \nu$ approaches the tensile saturation velocity $\nu_{t} \sim 2 \Delta x \tau_{n}^{j}(0)^{-1}$ while in the case of $\sigma_{11} \ll 0, \nu$ approaches the compressive velocity limit $\nu_{c} \sim \Delta x \tau_{n}^{i}(0)^{-1}$ and thus $\nu_{t} / \nu_{c} \sim 2$.

At present, it is unclear how the combined presence of dopants and stress alters growth kinetics. ${ }^{13-15}$ Furthermore, application of stress has been shown to cause significant alterations to the electronic structure of $\mathrm{Si}$ which, as per the GFLS model, may lead to synergistic dopant- and stressinfluenced SPEG kinetics. ${ }^{16-20}$ Thus, the goal of this work is to study the combined roles of electrically active impurities and stress on SPEG kinetics.

In this study, a polished 50-m-thick (001) Si wafer was $\mathrm{Si}^{+}$-implanted at 50,100 , and $200 \mathrm{keV}$ to doses of $1 \times 10^{15}$, $1 \times 10^{15}$, and $3 \times 10^{15} \mathrm{~cm}^{-2}$ and subsequently $\mathrm{B}^{+}$implanted at $60 \mathrm{keV}$ to a dose of $3.5 \times 10^{15} \mathrm{~cm}^{-2}$. The wafer was subsequently cleaved along $\langle 110\rangle$ directions into $\sim 0.2$ $\times 1.8 \mathrm{~cm}^{2}$ strips (with 1 and 2 directions taken to be [110] and $[1 \overline{1} 0]$ crystal directions) and uniaxially stressed up to magnitude of 1.0 GPa along [110] as presented elsewhere. ${ }^{21}$ The error in all nonzero stress measurements is estimated to be $\pm 0.1 \mathrm{GPa}$. Stress-free, tensilely stressed, and compres- 


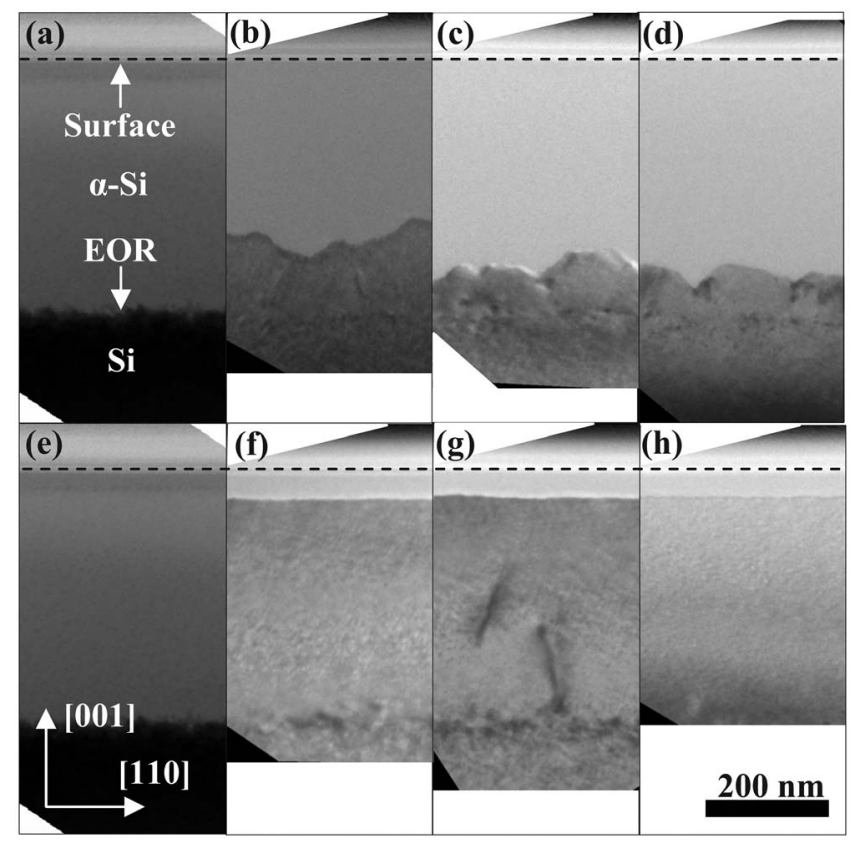

FIG. 1. [(a) and (e)] XTEM images of the as-implanted structure. XTEM images of specimens annealed for $7.0 \mathrm{~h}$ with applied stress of (b) $-0.25 \mathrm{GPa}$, (c) $-0.5 \mathrm{GPa}$, (d) $-1.0 \mathrm{GPa}$, (f) 0, (g) $0.5 \mathrm{GPa}$, and (h) $1.0 \mathrm{GPa}$.

sively stressed strips were annealed simultaneously at $500 \pm 1{ }^{\circ} \mathrm{C}$ in $\mathrm{N}_{2}$ ambient up to $11.2 \mathrm{~h}$. No detectable stress relaxation occurred during annealing. Growth was examined using cross-sectional transmission electron microscopy (XTEM). Approximately 70 XTEM specimens $\sim 10 \mu \mathrm{m}$ long were prepared via site-specific focused ion beam (FIB) milling within a distance of $\pm 3 \mathrm{~mm}$ from the strip centers to minimize the presence of any thermal gradient. Due to the very small specimen length to strip length ratio, it is reasonably assumed no intraspecimen stress gradients existed.

Figures 1(a) and 1(e) display XTEM micrographs of the as-implanted structure indicating an initial $\alpha$-Si layer $365 \pm 5 \mathrm{~nm}$ thick. The error in all $\alpha$-Si thickness (and $\mathrm{Si}$ growth) measurements is given as the root mean squared roughness of the $\alpha /$ crystalline interface. Annealing for $7.0 \mathrm{~h}$ with $\sigma_{11}=0$ resulted in $328 \pm 3 \mathrm{~nm}$ of growth with a planar $\alpha /$ crystalline interface, as shown in Fig. 1(f). End of range defects from ion implantation were present in all samples. In the case of annealing for $7.0 \mathrm{~h}$ with $\sigma_{11}=-0.25,-0.5$, and $-1.0 \mathrm{GPa}$, shown in Figs. 1(b)-1(d), $83 \pm 25,64 \pm 14$, and $57 \pm 10 \mathrm{~nm}$ of growth occurred which is less than the $\sigma_{11}$ $=0$ case. The growth interface was observed to roughen significantly with $\sigma_{11}<0$, presumably due to kinetically driven instabilities. ${ }^{11,13}$ In contrast, annealing with $\sigma_{11}=0.5$ and 1.0 GPa, shown in Figs. 1(g) and 1(h), produced nominally the same amount of growth as the $\sigma_{11}=0$ case. These observations are qualitatively consistent with recent studies of intrinsic stressed SPEG. ${ }^{11,12}$

The $\alpha$-Si thickness as a function of anneal time was measured for different $\sigma_{11}$ as shown in Fig. 2. The implanted $C_{\mathrm{B}}$ profile as measured using secondary ion mass spectrometry (SIMS) is superimposed in Fig. 2 indicating a peak $C_{\mathrm{B}}$ of $\sim 3.0 \times 10^{20} \mathrm{~cm}^{-3} \sim 200 \mathrm{~nm}$ deep. In cases of $0 \& \mathrm{nbsp}$; $\leqslant \sigma_{11} \leqslant 1.0 \mathrm{GPa}$, the $\alpha$-Si thickness versus time behavior was nominally the same for all $\sigma_{11}$ in this range and thus only the $\sigma_{11}=0$ set of data is reported for clarity. The growth kinetics for $\sigma_{11}<0$ were greatly retarded compared to the applied stress values $\left(\sigma_{11}\right.$ ).
Downloaded 30 Mar 2009 to 131.227 .178 .132 . Redistribution subject to AlP license or copyright; see http://apl.aip.org/apl/copyright.jsp

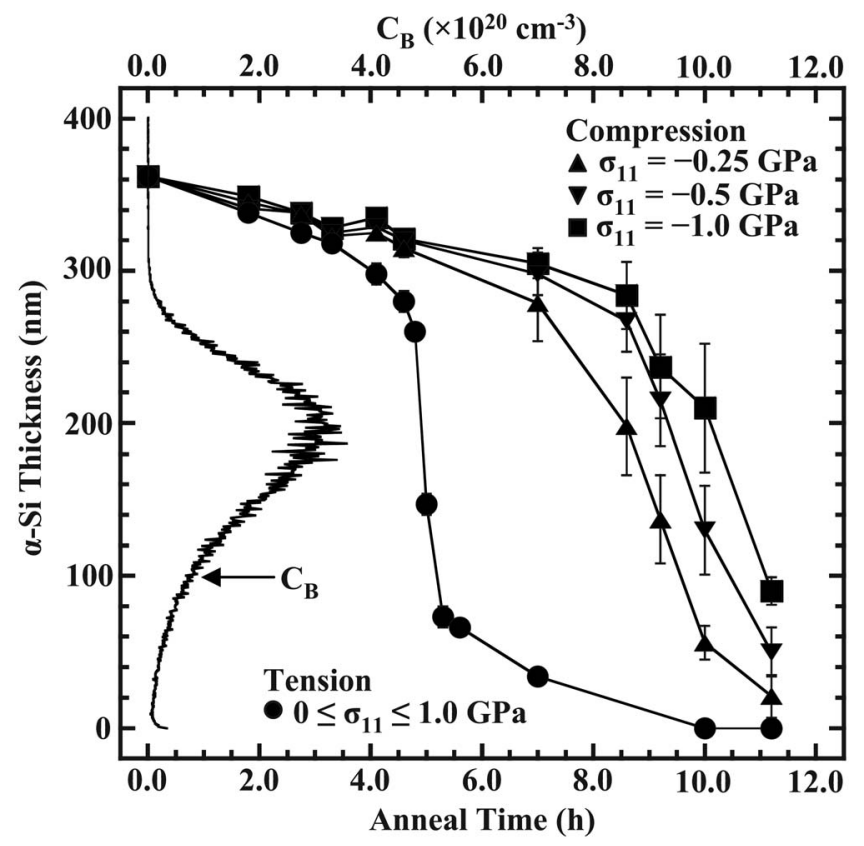

FIG. 2. $\alpha$-Si thickness versus anneal time behavior for different applied stress values $\left(\sigma_{11}\right)$ as superimposed on the SIMS-determined B concentration $\left(C_{\mathrm{B}}\right)$ profile.

$0 \leqslant \sigma_{11}$ cases. For all $\sigma_{11}$, the growth kinetics appears to vary with anneal time and increase with $C_{\mathrm{B}}$ as reported by others. $^{3-6}$

Figure 3 displays a plot of $\nu$ versus $C_{\mathrm{B}}$ for different $\sigma_{11}$ estimated from the data of Fig. 2 using the following method: (1) the average growth rate between two subsequent anneal times was calculated as the change in $\alpha$-Si thickness between the anneal times divided by the time interval (this is the reported $\nu$ ) and (2) the median value of $C_{\mathrm{B}}$ over the $\alpha$-Si thickness interval was obtained (this is the reported $C_{\mathrm{B}}$ ). For all $C_{B}, \nu$ was unchanged with $0<\sigma_{11}$ and retarded for -1.0 $\leqslant \sigma_{11} \leqslant-0.25 \mathrm{GPa}$. It is also evident from Fig. 3 that clear $\nu_{t}$ and approximate $\nu_{c}$ limits at a given $C_{\mathrm{B}}$ are observed as indicated.

Since $\nu$ versus $C_{\mathrm{B}}$ is constant for $0 \leqslant \sigma_{11}$, it is reasonable to extend Eq. (3) to $\nu=\nu_{t}$. Equation (3) was fit to the $\nu_{t}$ data

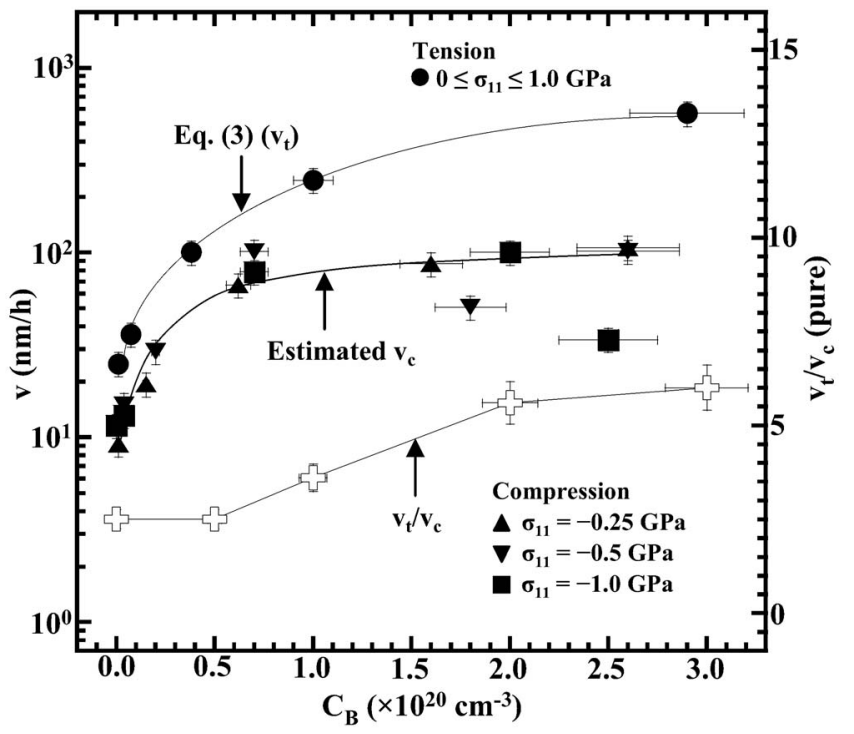

FIG. 3. Effect of B concentration $\left(C_{\mathrm{B}}\right)$ on growth velocity $(\nu)$ for different applied stress values $\left(\sigma_{11}\right)$. AIP license or copyright; see http://apl.aip.org/apl/copyright.jsp 
in Fig. 3 (assuming stress-independent $n_{i} \sim 10^{17} \mathrm{~cm}^{-3}$ ) producing $\tau_{n}^{i}(0)=(9.0 \pm 0.5) \times 10^{-3} \mathrm{~h}, \quad g=1.0 \pm 0.1$, and $\Delta E$ $=0.34 \pm 0.02 \mathrm{eV}$, where $\Delta E=E_{F}^{i}-E_{n}^{p}$. The values of $g$ and $\Delta E$ are in good agreement with those from the GFLS model. ${ }^{6}$ Thus, it appears $0<\sigma_{11}$ does not appreciably alter $E_{F}^{i}-E_{n}^{p}$. The results support the assumption of $n_{i}$ being stress independent. However, considering the vast body of prior work regarding stress-induced band structure changes, ${ }^{16-20}$ it may also be the case that application of $0<\sigma_{11}$ induces compensating alterations to $E_{F}^{i}$ and $E_{n}^{p}$ such that $E_{F}^{i}-E_{n}^{p}$ remains constant.

Figure 3 also displays the estimated $\nu_{t} / \nu_{c}$ versus $C_{\mathrm{B}}$ behavior. The ratio for lower $C_{\mathrm{B}}$ is near 2 but $\nu_{t} / \nu_{c} \sim 6$ as $C_{\mathrm{B}}$ increases past $\sim 1.5 \times 10^{20} \mathrm{~cm}^{-3}$. In the case of B-doped $\mathrm{Si}$ $\nu_{t} \sim 2 \Delta x \tau_{n}^{p}(0)^{-1}$ as nucleation kinetics do not appear to be influenced by $0<\sigma_{11}$. Thus, as per the observed $\nu_{t} / \nu_{c}$ values, $\nu_{c} \sim \Delta x \tau_{n}^{p}\left(\sigma_{11} \ll 0\right)^{-1}$ in B-doped Si where $3 \times \tau_{n}^{p}\left(\sigma_{11} \ll 0\right)^{-1}$ $\sim \tau_{n}^{p}(0)^{-1}$. In intrinsic SPEG, $\sigma_{11}$ does not alter nucleation kinetics as per the activation volume tensor for crystal island formation $\left(\Delta V_{i j}^{n}\right) .^{11,12}$ Presumably, $\Delta V_{i j}^{n}$ for charged nuclei is of the same form. Thus, an explanation for the retarded nucleation kinetics with $\sigma_{11} \ll 0$ is due to stress-induced changes in the Si band structure. Assuming the GFLS model is valid for in-plane compression, it therefore appears that $\sigma_{11}<0$ increases $n_{i}$ and/or increases $E_{F}^{i}-E_{n}^{p}$.

The results of this study suggest dopant and stress influences in SPEG may be synergistic. This is an important result as prior work of combined dopant- and stress-influenced SPEG assumed the two influences were independent. ${ }^{13-15}$ In particular, synergy would be important to consider in any SPEG simulations. ${ }^{22}$

Of course, there are several challenges in this work. Accurately characterizing $\nu$ as a function of $C_{\mathrm{B}}$ with a variable dopant profile is difficult, especially due to the ex situ nature of the experiments. Another issue is growth interface roughening with $\sigma_{11}<0$ which is partly stress driven, but is also dopant gradient driven. ${ }^{13}$ Prior work of intrinsic SPEG with $\sigma_{11} \ll 0$ observed roughening nearly an order of magnitude less than that observed herein. ${ }^{11,12}$ A possible way to avoid these issues in future work would be to use $\mathrm{Si}$ wafers with epitaxial layers with constant $C_{\mathrm{B}}$.

In summary, the influence of combined dopant- and stress-influenced SPEG of amorphized (001) Si was investigated. As per the GFLS model of dopant-enhanced SPEG, it appears stress may alter the Si electronic structure such that dopant and stress influences are synergistic in growth kinetics.

The authors acknowledge the Semiconductor Research Corporation (Task ID 1372.003) for funding this research. The Major Analytical Instrumentation Center at the University of Florida is acknowledged for use of the FIB and TEM facilities.

${ }^{1}$ G. L. Olsen and J. A. Roth, Mater. Sci. Rep. 3, 1 (1988).

${ }^{2}$ S. M. Hu, J. Appl. Phys. 70, R53 (1991).

${ }^{3}$ L. Csepregi, E. F. Kennedy, T. J. Gallagher, J. W. Mayer, and T. W. Sigmon, J. Appl. Phys. 48, 4234 (1977).

${ }^{4}$ J. S. Williams and R. G. Elliman, Phys. Rev. Lett. 51, 1069 (1983).

${ }^{5}$ J. C. McCallum, Nucl. Instrum. Methods Phys. Res. B 148, 350 (1999).

${ }^{6}$ B. C. Johnson and J. C. McCallum, Phys. Rev. B 76, 045206 (2007).

${ }^{7}$ M. A. Green, J. Appl. Phys. 67, 2944 (1990).

${ }^{8}$ F. Spaepen, Acta Metall. 26, 1167 (1978).

${ }^{9}$ J. S. Williams, R. G. Elliman, W. L. Brown, and T. E. Seidel, Phys. Rev. Lett. 55, 1482 (1985).

${ }^{10}$ P. A. M. Dirac, Proc. R. Soc. London, Ser. A 123, 714 (1929).

${ }^{11}$ N. G. Rudawski, K. S. Jones, and R. Gwilliam, Phys. Rev. Lett. 100, 165501 (2008)

${ }^{12}$ N. G. Rudawski, K. S. Jones, and R. Gwilliam, Mater. Sci. Eng., R. 61, 40 (2008).

${ }^{13}$ W. Barvosa-Carter and M. J. Aziz, Appl. Phys. Lett. 79, 356 (2001).

${ }^{14}$ W. Barvosa-Carter, M. J. Aziz, A.-V. Phan, T. Kaplan, and L. J. Gray, J. Appl. Phys. 96, 5462 (2004).

${ }^{15}$ N. G. Rudawski, K. S. Jones, and R. G. Elliman, J. Vac. Sci. Technol. B 26, 435 (2008).

${ }^{16}$ J. J. Wortman, J. R. Hauser, and R. M. Burger, J. Appl. Phys. 35, 2122 (1964).

${ }^{17}$ Y. Kanda, Jpn. J. Appl. Phys. 6, 475 (1967).

${ }^{18}$ C. G. Van de Walle and R. M. Martin, Phys. Rev. B 34, 5621 (1986).

${ }^{19}$ I. Balslev, Phys. Rev. 143, 636 (1966).

${ }^{20} \mathrm{C}$. Herring and E. Vogt, Phys. Rev. 101, 944 (1956)

${ }^{21}$ N. G. Rudawski, K. S. Jones, and R. Gwilliam, Appl. Phys. Lett. 91, 172103 (2007)

${ }^{22}$ S. Morarka, N. G. Rudawski, and M. E. Law, J. Vac. Sci. Technol. B 26, 357 (2008) 